\title{
EXIGÊNCIA MÍNINA DE FÓSFORO EM NOVILHOS DA RAÇA NELORE1
}

\author{
JOSÉ CLETO DA SILVA FILHO², DORINHA MIRIAM SILBER SCHIMIDT VITTI \\ OTÁVIO CAMPOS NETO ${ }^{4}$ e ADIBE LUIZ ABDALLA ${ }^{5}$
}

\begin{abstract}
RESUMO - A determinação da absorção real de fósforo (P) em bovinos deve levar em consideração a fração endógena mínina do mineral, que se perde nas fezes. De modo geral esses cálculos são feitos utilizando-se tabelas cujos valores foram obtidos em outros países, com outras raças de animais e em condições bem diferentes das condições brasileiras. $\mathrm{O}$ trabalho teve como objetivo determinar a perda endógena de $\mathrm{P}$ nas fezes e estimar a exigência mínima de $\mathrm{P}$ em novilhos da raça Nelore (Bos indicus). Foram utilizados 18 novilhos castrados, com peso médio de $190,82 \pm 27,53 \mathrm{~kg}$ e idade aproximada de 12 meses, divididos em três grupos de seis animais, e mantidos em gaiolas metabólicas individuais. Os animais receberam dieta básica constituída de feno de Brachiaria decumbens e uma mistura de concentrados durante os 30 dias de período experimental. Os tratamentos consistiram de diferentes quantidades de fosfato bicálcico em níveis de $0,12,0,24$ e $0,36 \%$ de P, com base na dieta total. Foram aplicadas injeções de ${ }^{32} \mathrm{P}$ nos animais para a determinação da perda endógena fecal de P. A perda endógena mínima fecal de P para uma ingestão zero do mineral, calculada por interpolação, foi de $5,72 \mathrm{mg} / \mathrm{kg}$ de peso vivo e para um balanço zero, o requerimento mínimo foi de $8,84 \mathrm{mg} / \mathrm{kg}$ de peso vivo por dia.
\end{abstract}

Termos para indexação: Bos indicus, fosfato bicálcico, digestibilidade, excreção, necessidades de nutrientes.

\section{PHOSPHORUS REQUIREMENT OF NELORE STEERS}

\begin{abstract}
The nutritional phosphorus (P) requirement of cattle has to consider the minimum endogenous fecal loss of this element. In general, ration formulation is based on foreign tables, without taking Brazilian conditions in consideration. The aim of this study was to determine the endogenous losses of $\mathrm{P}$ and to estimate the minimum $\mathrm{P}$ requirement of Nelore cattle. Eighteen castrated steers aged 12 months and weighing $190.82 \pm 27.53 \mathrm{~kg}$ were housed in metabolism cages. In a 30 day experimental period the animals were fed with hay and a concentrate mixture. Different levels of dicalcium phosphate were added to basal diet as to obtain $0.12,0.24$ and $0.36 \%$ of $\mathrm{P}$, based on the total dry matter intake. ${ }^{32} \mathrm{P}$ was intravenously injected and endogenous $\mathrm{P}$ losses were determined. The minimum endogenous fecal loss for zero P intake calculated by interpolation was $5.72 \mathrm{mg} / \mathrm{kg}$ (LW) per day, and for zero balance, the minimum P requirement was $8.84 \mathrm{mg} / \mathrm{kg}(\mathrm{LW})$ per day.
\end{abstract}

Index terms: Bos indicus, dicalcium phosphate, digestibility, excretion, nutritional requirements.

${ }^{1}$ Aceito para publicação em 10 de março de 2000. Financiado pela Fundação de Amparo à Pesquisa do Estado de São Paulo (FAPESP).

${ }^{2}$ Químico, Ph.D., Dep. de Zootecnia, Universidade Federal de Lavras (UFLA), Caixa Postal 37, CEP 37200-000 Lavras, MG. E-mail: cleto@ufla.br

${ }^{3}$ Biól., Ph.D., Centro de Energia Nuclear na Agricultura (CENA), Universidade de São Paulo (USP), Caixa Postal 96, CEP 13400-970 Piracicaba, SP. E-mail: dovitti@cena.usp.br

${ }^{4}$ Méd. Vet., Ph.D., Faculdade de Medicina Veterinária e Zootecnia, Universidade Estadual Paulista (UNESP), Caixa Postal 560, CEP 18618-000 Botucatu, SP.

${ }^{5}$ Eng. Agrôn., Ph.D., CENA, USP.

E-mail: abdalla@cena.usp.br

\section{INTRODUÇÃO}

Para determinar a quantidade mínima de fósforo (P) que determinada raça de bovinos necessita para se manter, é necessário conhecer também a quantidade mínima de $\mathrm{P}$ endógeno que o animal excreta. Esta excreção é considerada uma perda inevitável, e varia em razão do estado fisiológico do animal (Peeler, 1972). Challa \& Braithwaite (1988) demonstraram que a perda endógena de $\mathrm{P}$ em bovinos não é constante e está relacionada com o $\mathrm{P}$ consumido, representando, assim, um importante mecanismo na homeostase 
desse mineral. Estudos sobre perda endógena são indispensáveis para melhor compreensão do metabolismo de $\mathrm{P}$ e para os cálculos de requerimento dos animais. Conhecendo-se a exigência mínima de $\mathrm{P}$ do animal, pode-se alterar os níveis de suplementação recomendados.

Alguns trabalhos têm sido desenvolvidos, no Brasil, para obter informações que permitam fazer estimativas de exigências nutricionais nas condições brasileiras, adequando, assim, conhecimentos básicos oriundos de outros países para a nossa realidade (Silva, 1995).

O trabalho teve como objetivo determinar a perda endógena de $\mathrm{P}$ nas fezes e estimar a exigência mínima de $\mathrm{P}$ em bovinos da raça Nelore.

\section{MATERIAL E MÉTODOS}

Foram utilizados 18 novilhos castrados, da raça Nelore, divididos em três grupos de seis animais, com peso médio de $190,82 \pm 27,53 \mathrm{~kg}$ e idade aproximada de 12 meses e provenientes da Faculdade de Medicina Veterinária e Zootecnia de Botucatu, UNESP, SP. Os animais foram mantidos em gaiolas metabólicas individuais no biotério do Laboratório de Nutrição Animal do Centro de Energia Nuclear na Agricultura em Piracicaba, USP, SP, onde receberam uma dieta básica constituída de feno de Brachiaria decumbens e mistura de concentrados (Tabela 1), de acordo com as suas exigências (National Research Council, 1984) por um período de adaptação de 15 dias. Na fase experimental, com duração de 30 dias, além da dieta básica os animais receberam fosfato bicálcico como fonte de $\mathrm{P}$, para fornecer os níveis de $0,12,0,24$ e $0,36 \%$ de P, com base na dieta total, denominados Tratamento 1,2 e 3 ,

TABELA 1. Análise bromatológica da dieta.

\begin{tabular}{lcc}
\hline Componente $^{1}$ & Feno $(\%)$ & Concentrado $(\%)^{2}$ \\
\hline Matéria seca & 95,25 & 93,80 \\
Matéria mineral & 7,73 & 2,36 \\
Extrato etéreo & 1,80 & 1,04 \\
Fibra bruta & 33,24 & 6,04 \\
Proteína bruta & 7,25 & 19,44 \\
Fósforo inorgânico & 0,10 & 0,16 \\
\hline
\end{tabular}

1 Porcentagens calculadas em relação à matéria seca.

2 Concentrado: mistura de farelo de mandioca $(77,53 \%)$, farelo de soja $(19,38 \%)$, uréia $(2,62 \%)$ e mistura mineral $\left(2,16 \mathrm{~g}\right.$ de $\mathrm{CoSO}_{4}, 21,6 \mathrm{~g}$ de $\mathrm{CuSO}_{4}, 17,28 \mathrm{~g}$ de KI, $54 \mathrm{~g}$ de $\mathrm{FeSO}_{4}, 108 \mathrm{~g}$ de $\mathrm{ZnSO}_{4}, 91,8 \mathrm{~g}$ de $\mathrm{MgO}$ e $2160 \mathrm{~g}$ de $\mathrm{NaCl}$ ) respectivamente. Cada animal recebeu, em média, $2 \mathrm{~kg}$ de concentrado por dia. $\mathrm{O}$ fosfato bicálcico foi misturado ao concentrado.

No $20^{\circ}$ dia experimental, injetou-se na jugular direita de cada animal uma dose de $800 \mu \mathrm{Ci}$ de ${ }^{32} \mathrm{P}$, livre de carregador, e diluído em soro fisiológico. As coletas de sangue foram feitas a cada 24 horas, durante oito dias após a injeção, utilizando-se tubos a vácuo heparinizados. As fezes foram coletadas sempre no mesmo período e horário. Uma alíquota de $10 \%$ do total diário excretado foi armazenada em congelador para análises posteriores (Crampton \& Harris, 1969). O consumo de alimento e a excreção total de fezes foram registrados diariamente.

As amostras de sangue foram centrifugadas por $10 \mathrm{mi}-$ nutos, para separação do plasma. Foram precipitadas as proteínas em ácido tricloroacético a 10\%, e as determinações de P inorgânico nas amostras foram feitas por colorimetria (Fiske \& Subbarrow, 1925). As amostras de fezes foram maceradas, secadas $\left(100^{\circ} \mathrm{C}\right)$ e incineradas $\left(500^{\circ} \mathrm{C}\right)$. O teor de $\mathrm{P}$ inorgânico foi determinado pelo método de Sarruge \& Haag (1974). As determinações da radioatividade tanto no plasma como nas fezes foram feitas por efeito Cerenkov (International Atomic Energy Agency, 1979).

Os dados foram analisados utilizando-se o procedimento GLM do SAS (SAS Institute, 1986) considerandose um esquema em blocos casualizados. Pela análise de regressão foram determinados os valores de perda endógena mínima, e as exigências foram calculadas.

\section{RESULTADOS E DISCUSSÃO}

Não houve diferença significativa entre as médias dos pesos dos animais no final do experimento (Tabela 2).

Os valores médios do $\mathrm{P}$ consumido nos tratamentos 1 , 2 e 3 (Tabela 2) foram classificados, respectivamente, como baixo, adequado e excessivo (National Research Council, 1984). Challa \& Braithwaite (1988) consideraram nessa mesma ordem os níveis de 2,5, 6 e 10 g de P/dia, respectivamente. No presente experimento, os valores de $\mathrm{P}$ calculados em g/dia foram 5,05, 8,66 e 12,62, um pouco mais elevados que os obtidos por aqueles autores.

À medida que se elevou a ingestão de $\mathrm{P}$ suplementar, os níveis de $\mathrm{P}$ no plasma aumentaram (Tabela 2), observando-se uma relação linear entre $\mathrm{P}$ no plasma e $\mathrm{P}$ consumido $(\mathrm{P}<0,01)$ (Fig. 1). Os valores obtidos situam-se dentro dos limites considerados normais, ou seja, entre 4 e 9 mg/\% (Thompson Junior, 
TABELA 2. Parâmetros observados e relacionados ao metabolismo do fósforo.

\begin{tabular}{lccc}
\hline Observações & Tratamento 1 & Tratamento 2 & Tratamento 3 \\
\hline Número de animais & 6 & 6 & 6 \\
Peso dos animais (kg) & $193,40 \pm 13,40$ & $197,10 \pm 42,68$ & $182,40 \pm 24,03$ \\
Consumo de P (mg/kg PV) & $26,11 \pm 2,89$ & $43,93 \pm 6,64$ & $69,19 \pm 3,92$ \\
Excreção de P (mg/kg PV) & $23,23 \pm 1,84$ & $32,21 \pm 13,61$ & $44,10 \pm 9,87$ \\
P no plasma (mg/\%) & $6,16 \pm 0,65$ & $7,79 \pm 1,24$ & $9,06 \pm 1,81$ \\
P absorvido (mg/kg PV) & $15,28 \pm 2,62$ & $31,67 \pm 10,35$ & $47,38 \pm 6,95$ \\
P endógeno (mg/kg PV) & $12,39 \pm 2,98$ & $19,95 \pm 11,54$ & $22,29 \pm 6,16$ \\
Disponibilidade biológica de P & $55,41 \pm 12,60$ & $70,20 \pm 11,23$ & $68,57 \pm 9,97$ \\
\hline
\end{tabular}

${ }^{1}$ Tratamento 1: $0,12 \%$ de P; tratamento 2: 0,24\% de P; tratamento 3: $0,36 \%$ de P.

1978). Embora o teor de P no plasma não seja considerado isoladamente um bom parâmetro para avaliar o status nutricional de $\mathrm{P}$, os dados do presente experimento sugerem o contrário, mostrando a relação entre consumo do elemento e seus teores no plasma.

Os dados de excreção de $\mathrm{P}$ nas fezes convertidos em g/dia resultam, em média, em 4,50, 6,35 e 8,05, respectivamente, nos tratamentos 1,2 e 3 (Tabela 2 ). A suplementação de $\mathrm{P}$ causou um aumento na excreção do $\mathrm{P}$ fecal, linear com o $\mathrm{P}$ consumido $(\mathrm{P}<0,001)$ (Fig. 1). O aumento da excreção fecal de $\mathrm{P}$ com o consumo desse mineral em ruminantes foi reportado por vários autores (Scott et al., 1985; Challa \& Braithwaite, 1988; Zahari et al., 1994).

Os valores de $\mathrm{P}$ fecal obtidos no presente trabalho são mais elevados do que os observados na literatura. Em uma mesma faixa de consumo, Silva Filho et al. (1992) obtiveram, em gado mestiço de zebu, valores de excreção de $\mathrm{P}$ fecal em torno de $7 \mathrm{~g} /$ dia. Verificou-se que os valores de $\mathrm{P}$ nas fezes foram altos em relação ao $\mathrm{P}$ consumido. Deve ser ressaltado que esse valor inclui, além do $\mathrm{P}$ proveniente da dieta e não absorvido, a perda endógena de $\mathrm{P}$, ou seja, a quantidade de $\mathrm{P}$ que foi absorvida, metabolizada e finalmente excretada nas fezes (Georgievskii, 1982). De acordo com Peeler (1972), essas perdas são inevitáveis e são decorrentes do estado fisiológico, idade, sexo do animal, bem como da fonte de $\mathrm{P}$.

Os valores do $\mathrm{P}$ endógeno nas fezes aumentaram com os níveis de $\mathrm{P}$ oferecido na dieta (Tabela 2) e esta correlação foi expressa pela equação: $\mathrm{P}_{\text {endógeno }}=5,72+0,27 \mathrm{P}_{\text {consumido }}(\mathrm{P}<0,01 ; \mathrm{r}=0,60)$ (Fig. 1). Dados de perda endógena fecal são escas- sos em bovinos, porém os resultados obtidos estão próximos dos valores reportados por Silva Filho et al. (1992). De acordo com o Agricultural Research Council (1965), num bovino de $200 \mathrm{~kg}$ de peso vivo, a perda endógena fecal está em torno de $17 \mathrm{mg} / \mathrm{kg} / \mathrm{dia}$, considerando que essa perda não é influenciada pelo consumo de P. Entretanto, vários trabalhos mostram que há uma relação entre $\mathrm{P}$ endógeno fecal e o consumo de P (Braithwaite \& Labourne, 1984; Agricultural and Food Research Council, 1991; Coates \& Ternouth, 1992; Louvandini \& Vitti, 1994).

Em pesquisa desenvolvida em Viçosa, MG, com bovinos Nelore, Ezequiel (1987) obteve, em relação ao $\mathrm{P}$ endógeno, o valor de $17,44 \mathrm{mg} / \mathrm{kg}$ de PV/dia. Neste trabalho, os cálculos da perda endógena fecal foram feitos pela equação de regressão entre as quantidades de $\mathrm{P}$ excretado e a ingestão do elemento.

Challa \& Braithwaite (1988), em experimentos com bezerros, demonstraram que a perda endógena fecal não se manteve constante e foi correlacionada com o nível de ingestão de P. Com relação aos consumos de 2,5, 6 e $10 \mathrm{~g}$ de P/dia, os valores diários de $\mathrm{P}$ endógeno foram 12,06, 18,31 e 22,75 mg/ $\mathrm{kg}$ de $\mathrm{PV} / \mathrm{dia}$; valores esses bem próximos dos obtidos no presente experimento. $\mathrm{O}$ aumento da perda endógena com o nível de $\mathrm{P}$ na dieta deve-se, em parte, à maior secreção via saliva. De acordo com o Agricultural and Food Research Council (1991), não há evidências de que ocorra um mecanismo de saturação na secreção salivar de P. Entretanto, Louvandini (1995) observou, em ovinos, que houve uma estabilização nos valores do $\mathrm{P}$ salivar e endógeno, com um consumo de $\mathrm{P}$ acima de $100 \mathrm{mg} / \mathrm{kg}$ de $\mathrm{PV} / \mathrm{dia}$. No 

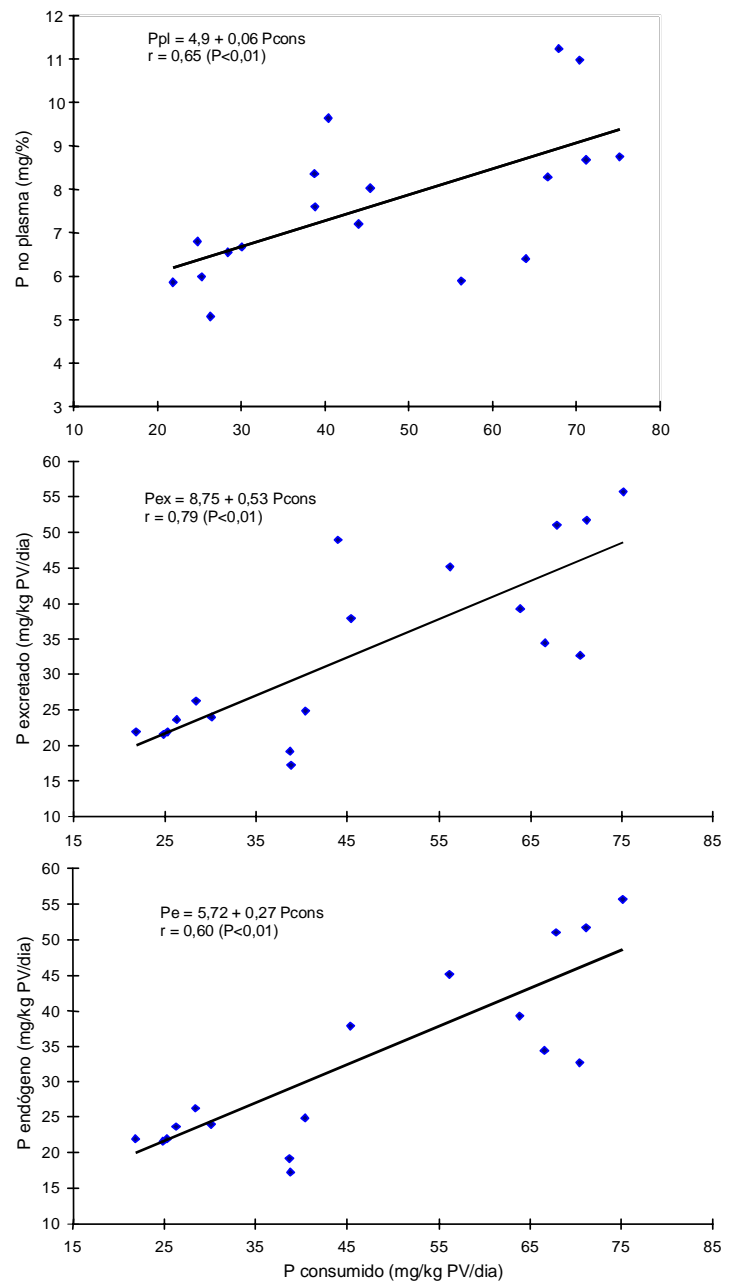

FIG. 1. Fósforo no plasma (Ppl), fósforo excretado (Pex) e fósforo endógeno (Pe) em função do fósforo consumido (Pcons).

presente experimento, o maior nível de ingestão foi de $69 \mathrm{mg} / \mathrm{kg}$ de PV/dia, e portanto não atingiu um valor excessivo.

Através da equação que mostra a relação $P$ endógeno:P consumido, quando se considera um consumo de P igual a zero, obtém-se o valor da perda endógena mínima de 5,72 mg/kg de PV/dia. Este valor está um pouco abaixo do valor citado por Challa et al. (1989) de $8,6 \mathrm{mg} / \mathrm{kg}$ de PV/dia e pelo Agricultural Research Council (1980), de 10 mg/kg de PV/dia. Como no presente trabalho a perda urinária de $\mathrm{P}$ não foi quantificada, por ser insignificante em ruminantes, o valor de $5,72 \mathrm{mg} / \mathrm{kg}$ de PV/dia representa a quantidade que os animais devem absorver para suprir as perdas inevitáveis. Para converter a perda endógena mínima em requerimento de mantença, pode-se utilizar o valor da absorção verdadeira ou coeficiente de absorção médio de $64,72 \%$, obtido neste trabalho. Assim, o requerimento dietético diário, em bovinos, nas condições experimentais, é de $8,84 \mathrm{mg} / \mathrm{kg}$ de PV/dia. Pires (1991) cita que a exigência total de $\mathrm{P}$ em bovinos Nelore com peso em torno de $200 \mathrm{~kg}$ é de $12,17 \mathrm{mg} / \mathrm{kg}$ de $\mathrm{PV} / \mathrm{dia}$, valor este maior que o obtido no presente experimento. Esse autor utilizou, para os cálculos da exigência, os valores de perda endógena fecal e de disponibilidade baseados no Agricultural Research Council (1980).

Challa \& Braithwaite (1988) obtiveram, em bezerros em crescimento, um valor da perda endógena mínima de $\mathrm{P}$ de $8,34 \mathrm{mg} / \mathrm{kg}$ de $\mathrm{PV} /$ dia. Considerando o valor da eficiência de absorção obtida por esses autores, de $70 \%$, verifica-se um valor de $11,9 \mathrm{mg} / \mathrm{kg}$ de PV/dia como o requerimento dietético em bezerros. Este dado é um pouco mais elevado que o observado no presente trabalho. Deve-se levar em conta que vários fatores podem influenciar esses parâmetros, destacando-se a espécie, a raça, o clima e fatores geográficos.

$\mathrm{Na}$ literatura, praticamente não existem dados de perda endógena e eficiência de absorção de P relativos a bovinos da raça Nelore.

\section{CONCLUSÕES}

1. Novilhos da raça Nelore com pesos próximos de $200 \mathrm{~kg}$ (peso vivo) apresentam uma perda endógena fecal mínima de $\mathrm{P}$ em torno de $5,72 \mathrm{mg} / \mathrm{kg}$ de peso vivo.

2. Considerando a disponibilidade biológica do P do fosfato bicálcico em $64,72 \%$, esses animais têm uma exigência líquida diária de $\mathrm{P}$ de $8,84 \mathrm{mg} / \mathrm{kg}$ de peso vivo em regime de mantença.

\section{REFERÊNCIAS}

AGRICULTURAL AND FOOD RESEARCH COUNCIL. Technical Committee on Responses to Nutrients. A Reappraisal of the calcium and phosphorus requirements of sheep and cattle. Nutrition Abstracts and Reviews: Series B Livestock Feeds and Feeding, Wallingford, v.61, n.9, p.573-612, 1991. 
AGRICULTURAL RESEARCH COUNCIL (Londres, Inglaterra). The nutrient requirement of farm livestock. London : Commonwealth Agricultural Bureau, 1965. p.14-84.

AGRICULTURAL RESEARCH COUNCIL (Londres, Inglaterra). The nutrient requirement of ruminant livestock. London : Commonwealth Agricultural Bureau, 1980.351p.

BRAithwaite, G.D.; LABOURNE, L.J. Some observations of phosphorus homeostasis and requirements of sheep. Journal of Agricultural Science, Cambridge, Grã-Bretanha, v.102, p.295306, 1984.

CHALLA, J.; BRAITHWAITE, G.D. Phosphorus and calcium metabolism in growing calves with special emphasis on homeostasis. 1. Studies of the effect of changes in the dietary phosphorus intake and calcium metabolism. Journal of Agricultural Science, Cambridge, Grã-Bretanha, v.110, n.3, p.573-581, 1988.

CHALLA, J.; BRAITHWAITE, G.D.; DHANOA, M.S. Phosphorus homeostasis in growing calves. Journal of Agricultural Science, Cambridge, Grã-Bretanha, v.112, p.217-226, 1989.

COATES, D.B.; TERNOUTH, J.H. Phosphorus kinetics of cattle grazing tropical pastures and implications for estimation of their phosphorus requirements. Journal of Agricultural Science, Cambridge, GrãBretanha, v.119, n.3, p.401-410, 1992.

CRAMPTON, E.W.; HARRIS, L.E. The proximate analysis of feeds. In: CRAMPTON, E.W.; HARRIS, L.E. Applied animal nutrition. 2.ed. San Francisco : W.E. Freeman, 1969. p.30-55.

EZEQUIEL, J.M.B. Exigências de proteína e minerais de bovídeos: frações endógenas. Viçosa : UFV-Imprensa Universitária, 1987. 131p. Tese de Doutorado.

FISKE, C.H.; SUBBARROW, Y. The colorimetric determination of phosphorus. Journal of Biological Chemistry, Bethesda, v.66, n.2, p.375-400, 1925.

GEORGIEVSKII, V.I. The physiological role of macroelements. In: GEORGIEVKII, V.I.; ANNENKOV, B.N.; SAMOKHIN, V.I. Mineral nutrition of animals. London : Butterworths, 1982. p.91-170.

INTERNATIONAL ATOMIC ENERGY AGENCY (Viena, Austria). Laboratory training manual on the use of nuclear techniques in animal research. Vienna, 1979. 299p. (Technical Report Series, 193).

LOUVANDINI, H. Perda endógena de fósforo em ovinos suplementados com diferentes níveis do elemento na dieta. São Paulo : Instituto de Pesquisas Energéticas e Nucleares, 1995. 87p. Tese de Doutorado.
LOUVANDINI, H.; VITTI, D.M.S.S. Perda endógena de fósforo em ovinos, com diferentes níveis do elemento na dieta. Pesquisa Agropecuária Brasileira, Brasília, v.29, n.1, p.145-149, jan. 1994.

NATIONAL RESEARCH COUNCIL (Washington, Estados Unidos). Nutrient requirement of beef cattle. 6.ed. Washington : National Academy of Sciences, 1984. 90p.

PEELER, H.T. Biological availability of nutrients in feeds: availability of major mineral ions. Journal of Animal Science, Champaign, v.35, n.3, p.695-712, 1972.

PIRES, K.C. Exigências de proteína, energia e macroelementos minerais ( $\mathrm{Ca}, \mathrm{P}, \mathrm{Mg}, \mathrm{K}$ e $\mathrm{Na}$ ) de bovinos não-castrados de três grupos genéticos. Viçosa : UFV- Imprensa Universitária, 1991. 125p. Tese de Doutorado.

SARRUGE, J.R.; HAAG, H.P. Determinação colorimétrica do fósforo. In: SARRUGE, J.R.; HAAG, H.P. Análises químicas em plantas. Piracicaba : ESALQ, 1974. p.6-58.

SAS INSTITUTE (Cary, Estados Unidos). SAS system for linear models. Cary, 1986. 211p.

SCOTT, D.; WHITELAW, F.G.; BUCHAN, W.; BRUCE, L.A. The effect of variation in phosphorus intake on salivary phosphorus secretion, net phosphorus absorption and faecal endogenous excretion in sheep. Journal of Agricultural Science, Cambridge, GrãBretanha, v.105, p.271-277, 1985.

SILVA, J.F. C. da. Exigências de macroelementos inorgânicos para bovinos: o sistema ARC/AFRC e a experiência no Brasil. In: SIMPÓSIO INTERNACIONAL SOBRE EXIGÊNCIAS NUTRICIONAIS DE RUMINANTES, 1995, Viçosa. Anais. Viçosa : UFV, 1995. p.1467-1504.

SILVA FILHO, J.C.; LOPES, H.O.S.; MEIRELLES, C.F.; VITTI, D.M.S.S.; ABDALLA, A.L. Absorção real do fósforo do fosfato bicálcico, fosfato monoamônio, superfosfato triplo e do fosfato de uréia em bovinos. Pesquisa Agropecuária Brasileira, Brasília, v.27, n.1, p.1-6, jan. 1992.

THOMPSON JUNIOR, W.R. Phosphorus in animal nutrition. In: PHOSPHORUS for agriculture: a situation analysis. Atlanta : Potash \& Phosphate Institute, 1978. p.126-158,

ZAHARI, W.M.; SCOTT, D.; LOVERIDGE, N.; BUCHAN, W.; MILNE, J. The effect of high phosphorus intake on calcium and phosphorus retention and bone turnover in growing lambs. Experimental Physiology, Cambridge, Grã-Bretanha, v.79, p.175-181, 1994.

Pesq. agropec. bras., Brasília, v.35, n.9, p.1861-1865, set. 2000 